\title{
Eficácia do óleo de citronela [Cymbopogon nardus (L.) Rendle] no controle de ectoparasitas de bovinos
}

\author{
AGNOLIN, C.A. ${ }^{1 *}$; OLIVO, C.J. ${ }^{1}$; LEAL, M.L.R. ${ }^{2}$; BECK, R.C.R. ${ }^{3}$; MEINERZ, G.R. ${ }^{1}$; PARRA, C.L.C. ${ }^{1}$; MACHADO, \\ P.R. ; FOLETTO, V. ; BEM, C.M. ${ }^{1}$; NICOLODI, P.R.S.J. ${ }^{4}$ \\ 'Universidade Federal de Santa Maria, Centro de Ciências Rurais, Departamento de Zootecnia \\ *caiozoot2002@yahoo.com.br ${ }^{2}$ Departamento de Clínica de Grandes Animais ${ }^{3}$ Departamento de Farmácia Industrial, \\ CEP: 97105-900, Santa Maria-Brasil ${ }^{4}$ Mestre em Medicina Veterinária, Universidade Federal de Santa Maria, CEP: \\ 97105-900, Santa Maria-Brasil
}

\begin{abstract}
RESUMO: O objetivo desta pesquisa foi avaliar o efeito in vivo do óleo de citronela, no controle do carrapato bovino [Rhipicephalus (Boophilus) microplus], da mosca-dos-chifres (Haematobia irritans), da mosca-dos-estábulos (Stomoxys calcitrans) e da mosca doméstica (Musca domestica). Foram utilizadas 15 vacas da raça Holandês, distribuídas em três grupos de cinco animais cada um. Os tratamentos foram: controle negativo, amitraz a $0,025 \%$ e óleo de citronela a $4 \%$. Para avaliação foram contadas fêmeas ingurgitadas de carrapato e moscas antes (média dos dias - $3,-2,-1$ ) e após a aplicação dos produtos nos dias 7, 14, 21 e 28; também foram coletadas amostras de sangue. Em 28 dias, houve necessidade de se reaplicar o amitraz e o fitoterápico para controlar a infestação com carrapato. A relação entre o número de aplicações foi de 1:2,5 para o amitraz e o óleo de citronela, respectivamente. A eficácia no controle do carrapato foi de 71,8 e 30,9\% para o amitraz e óleo de citronela a 4\%, respectivamente, na média pós-tratamento. Verificou-se baixo controle de moscas no tratamento constituído pelo fitoterápico. Não houve diferença entre os tratamentos para os parâmetros sanguíneos.
\end{abstract}

Palavras-chave: acaricida, bovino leiteiro, fitoterápico, óleo essencial

\begin{abstract}
Efficacy of citronella [Cymbopogon nardus (L.) Rendle] oil in the control of bovine ectoparasites. This study aimed to evaluate the in vivo effect of citronella oil on the control of bovine ticks [Rhipicephalus (Boophilus) microplus], horn flies (Haematobia irritans), stable flies (Stomoxys calcitrans) and houseflies (Musca domestica). Fifteen Holstein cows were allocated to three groups of five animals each. The treatments were: negative control, amitraz at $0.025 \%$ and citronella oil at $4 \%$. Engorged female ticks and flies were counted before (mean of days $-3,-2,-1)$ and at $7,14,21$ and 28 days after treatment; blood samples were also collected. Within 28 days, amitraz and the phytotherapic agent had to be reapplied to control tick infestation. The relationship among the number of applications was 1:2.5 for amitraz and citronella oil, respectively. The efficacy of tick control was, on average, 71.8 and $30.9 \%$ for amitraz and citronella oil at $4 \%$ respectively, post-treatment. Lower control of flies was observed for the phytotherapic group. There was no difference among treatments for blood parameters.
\end{abstract}

Key words: acaricide, dairy cattle, phytotherapic agent, essential oil

\section{INTRODUÇÃO}

Um dos fatores mais importantes na diminuição da eficiência produtiva dos bovinos é o ectoparasitismo (Bianchin et al.,1999). Dentre os principais parasitas externos, o carrapato [Rhipicephalus (Boophilus) microplus] é o que apresenta maior impacto econômico, não somente pela espoliação que provoca, mas também pela transmissão de doenças aos animais (Johnston \& Haydock, 1969), embora outros como a mosca-dos-chifres, a moscados-estábulos e a mosca doméstica apresentam importância significativa no rebanho bovino leiteiro (Grisi et al., 2002). Anualmente os produtores brasileiros gastam cerca de $R \$ 800$ milhões de reais com produtos químicos no controle de parasitas

Recebido para publicação em 11/05/2009

Aceito para publicação em 16/04/2010

Rev. Bras. PI. Med., Botucatu, v.12, n.4, p.482-487, 2010. 
(Camillo et al., 2009).

Como a erradicação do carrapato não é uma estratégia para o controle das infestações (Cordovés, 1997), o uso de produtos inseticidas associados à rotação de pastagens, vacinas e criação de raças de bovinos com maior grau de resistência são frequentemente relatados como métodos mais eficientes de controle desse ectoparasita (Radostits et al., 2002). Mesmo com essas recomendações, o uso indiscriminado e inadequado de ectoparasiticidas químicos é prática corrente, implicando no desenvolvimento de resistência ao composto utilizado, notadamente para o carrapato (Oliveira \& Azevedo, 2002) e mosca-dos-chifres (Barros, 2001). Nessa estratégia deve-se considerar que parte dos produtores não observa os períodos de carência dos produtos utilizados (Heimerdinger et al., 2006).

Mesmo com a utilização de produtos acaricidas sintéticos em conjunto com outras estratégias de controle, a presença destes produtos na propriedade e a frequente utilização no rebanho são potenciais fontes de contaminação dos animais e, em consequência, do leite e da carne oriundas destes rebanhos (Dubois, 1993; Chagas et al., 2003). Como forma de redução do impacto ambiental e financeiro do uso destes acaricidas sintéticos, Castrejón et al. (2003) sugerem que sejam agregados métodos de controle não-químicos, como o uso de plantas com propriedades ectoparasiticidas. Nesse contexto, a fitoterapia pode ser ferramenta importante no controle dos ectoparasitas, possibilitando a associação com outras estratégias de controle e resultando num produto (carne e ou leite) livre de substâncias químicas que possam prejudicar 0 homem, o animal e o ambiente.

Dentre as plantas indicadas para o controle de insetos, destaca-se a citronela [Cymbopogon nardus (L.) Rendle], que é uma planta da família Poaceae, originária da ilha de Java na Indonésia e cultivada em regiões tropicais e subtropicais. O óleo, extraído das folhas, frescas ou parcialmente dessecadas, é usado como repelente de mosquitos. Essa propriedade é atribuída à presença de substâncias voláteis nas folhas, como citronelal, eugenol, geramiol e limoneno, entre outras, denominadas de um modo geral como monoterpenos (Shasany et al., 2000). Pesquisas conduzidas com o óleo dessa planta demonstraram a ação como inseticida e de repelência contra mosquitos e moscas (Raja et al., 2001), inibição da postura e eclosão dos ovos de carrapato (Martins, 2006).

O objetivo do presente trabalho foi determinar a quantidade de banhos necessários para o controle do carrapato utilizando um produto fitoterápico (óleo de citronela), em comparação a carrapaticida convencional (Amitraz).

\section{MATERIAL E MÉTODO}

O experimento foi realizado no Laboratório de Bovinocultura de Leite, pertencente ao Departamento de Zootecnia da Universidade Federal de Santa Maria, de janeiro a fevereiro de 2007. Foram constituídos três tratamentos, o Amitraz, a 0,025\% (grupo controle positivo), óleo de citronela a $4 \%$ e testemunha (grupo controle negativo).

Aopção pelo Amitraz deveu-se por não haver resistência da cepa utilizada ao referido produto químico. Para a concentração de óleo de citronela, o valor de $4 \%$ baseou-se em experimentações in vitro (Olivo et al., 2008). O óleo de citronela testado foi produzido na mesorregião Noroeste do Rio Grande do Sul, no Pólo Oleoquímico de Três Passos, sendo obtido da parte aérea de plantas frescas, mediante processo de destilação a vapor, apresentando rendimento de $0,7 \%$, aproximadamente. Os valores da análise cromatográfica apresentaram como componentes ativos mais importantes o citronelal, o geraniol e o citronelol, correspondendo a 50,07; 13,87; 7,93\%, respectivamente.

Nas avaliações foram utilizadas 15 vacas em lactação da raça Holandês malhada de preto, com cerca de $515 \mathrm{~kg}$ de peso vivo e produção média de 17 $\mathrm{kg}$ de leite/vaca/dia. Os animais eram submetidos diariamente a duas ordenhas. A base da alimentação era constituída por pastagens perenes de ciclo estival. A complementação alimentar, feita com concentrado (18\% PB), à razão de $4 \mathrm{~kg}_{\text {vaca-1 }} \mathrm{dia}^{-1}$, era dividida entre as ordenhas da manhã e da tarde.

Foram utilizadas cinco vacas por tratamento. O critério para utilização de cada animal foi a infestação de carrapatos (média de três dias consecutivos), sendo usadas vacas que apresentavam no mínimo dez teleóginas (fêmeas de carrapato adulto). Concomitantemente foram feitas contagens de moscas em todo animal.

As formulações foram feitas momentos antes da aplicação, sendo aplicadas após a ordenha da tarde, utilizando-se pulverizador costal. Para a elaboração da solução com o produto fitoterápico (óleo de citronela a $4 \%$ ) adicionou-se $0,5 \%$ de detergente neutro. A quantidade de calda usada foi de três litros/ vaca em ambos os tratamentos. A reaplicação dos produtos foi feita quando os animais apresentavam reinfestação mínima de 10 teleóginas por animal.

Para as avaliações foram efetuadas contagens de carrapato considerando-se os ínstares com tamanho superior a $4,5 \mathrm{~mm}$ de comprimento, na metade do corpo do animal (no caso, sempre o lado direito), multiplicando-se o valor por dois para a obtenção da infestação total (Wharton et al., 1970). A contagem de dípteros foi visual, quando os animais encontravam-se na mangueira de espera, antecedendo a ordenha, sendo feita sempre pelo mesmo avaliador e realizada a uma distância de 1,5

Rev. Bras. Pl. Med., Botucatu, v.12, n.4, p.482-487, 2010. 
$\mathrm{m}$ do animal, evitando-se, assim, o vôo dos insetos. Considerando-se as características do pelame e a dificuldade de se diferenciar a mosca doméstica da mosca-dos-estábulos fez-se a contagem conjunta desses dípteros. Para mosca-dos-chifres fez-se a contagem individual. A contagem de dípteros foi feita apenas nos grupos constituídos pelos tratamentos testemunha e fitoterápico, considerando-se que o produto químico não tem ação mosquicida.

Para calcular a eficácia do produto no controle dos ectoparasitas foi utilizada a seguinte fórmula: Eficácia $=[$ (№ de teleóginas de prétratamento - № de teleóginas do dia de póstratamento) X 100 / № de teleóginas de prétratamento].

Foram realizadas coletas de sangue (com EDTA) dos animais antes e em 72 e 120 horas após o primeiro banho. Estas amostras foram obtidas por punção da veia jugular, após tricotomia e antissepsia. As amostras foram destinadas à realização do hemograma completo: número de hemácias, volume globular, hemoglobina, volume corpuscular médio, concentração de hemoglobina corpuscular média, leucócitos, linfócitos, neutrófilos segmentados, neutrófilos bastonetes, eosinófilos e monócitos, mediante técnicas descritas por Feldman et al. (2000).

$O$ delineamento experimental utilizado foi o inteiramente casualizado, com cinco repetições (animais) por tratamento. Os resultados foram submetidos à análise de variância, ao nível de $5 \%$ de probabilidade do erro. Para a comparação entre médias usou-se o teste de Bonferroni. Também foi realizada análise de regressão polinomial, em função dos dias de contagens dos parasitas após a aplicação dos produtos. As análises foram realizadas com auxílio do programa estatístico SAS versão 6.1 (1997). O modelo matemático referente à análise das variáveis estudadas foi representado por: $Y_{i j k}=\mu+T_{i}+R_{j}\left(T_{i}\right)+$ $D_{k}+T D_{i k}+e_{i j k}$, em que, $Y_{i j k}$ representa a variável dependente; $i$ = índice de tratamentos; $j$ = índice de repetições; $\mathrm{k}$ = índice de dias de avaliação; $\mu$ é a média de todas as observações; $T_{i}$ corresponde ao efeito dos tratamentos; $R_{i}\left(T_{i}\right)$ é o efeito da repetição dentro do tratamento (erro a); $D_{k}$ é o efeito dos dias de avaliação; $T D_{i k}$ representa a interação entre os tratamentos e dias de avaliação; $\mathrm{e}_{\mathrm{ijk}}$ é o erro experimental residual (erro b).

\section{RESULTADO E DISCUSSÃO}

Os resultados obtidos (Tabela 1) demonstram que a relação de banhos entre o produto químico e o fitoterápico foi de dois para cinco $(1: 2,5)$, respectivamente. Observa-se que no grupo controle positivo, com amitraz, a necessidade de novo controle do carrapato foi de 28 dias. Resultados obtidos por Mekonnen (2001), que observou nova infestação 21 dias após aplicação do amitraz, também confirmam a menor eficácia desse carrapaticida sobre as formas inferiores do carrapato, considerando que ele pode permanecer até 35 dias no bovino (Cordovés, 1997). Os dados de eficácia do amitraz (Figura 1), submetidos à análise de regressão, demonstram comportamento cúbico $(p<0,05)$, com início ascendente, indicando elevado controle no início do tratamento.

Para o grupo tratado com óleo de citronela, verificou-se diferença $(p<0,05)$ quanto ao número de teleóginas entre o pré-tratamento e as demais avaliações. Comparando-se com o amitraz, observase que a solução constituída pelo fitoterápico controlou parcialmente o carrapato, embora maior freqüência de banhos (nos 7, 14, 21 e 28 dias pós-tratamento) foi necessária para se obter esse desempenho. Resultados obtidos com avaliações in vitro com 3\% de óleo de citronela, mediante três aplicações de 1\% em três dias consecutivos, em dois experimentos, nos quais se testou diferentes origens do produto, verificaram-se eficácias bem superiores, sendo de 79,3 e de $92,1 \%$ (Olivo et al., 2008).

Destaca-se que no grupo constituído por óleo de citronela a infestação foi mantida sob controle com número de teleóginas sempre abaixo do valor do prétratamento, enquanto que no controle negativo houve aumento de superior a $500 \%$ aos 28 dias (Tabela 1), verificando-se comportamento linear ascendente (Figura 1). A ação acaricida do óleo de citronela devese, provavelmente, ao princípio ativo citronelal (Chagas et al., 2002), que é o principal componente desse produto. Pesquisa conduzida com citronelal, geraniol e citronelol, estudados separadamente, confirmou efeito significativamente maior $(p<0,05)$ do citronelal e do geraniol em relação ao citronelol, com ação larvicida e acaricida, controlando teleóginas com inibição da postura e da eclodibilidade dos ovos (Martins, 2006).

A ação acaricida do óleo de citronela também foi comprovada por Chungsamarnyart \& Jiwajinda (1992) que, utilizando soluções contendo 7,1; 8,3 e $12,5 \%$ de óleo diluído em etanol verificaram efeito larvicida de 58,1; 92,7 e 95,7\% respectivamente. Martins (2006), usando concentrações de 4,1 e 6,1\%, verificou um controle próximo a $50 \%$ para larvas e teleóginas. Outro aspecto que se deve considerar, embora não avaliado no presente trabalho, é o efeito do produto sobre a inibição da postura das teleóginas, condição essa comprovada ao se usar formulações com $10 \%$ de óleo de citronela ou em concentrações com 7,5\% que inibe a eclosão das larvas (Martins, 2006). Mesmo considerando-se que o nível usado foi mais baixo, é possível que o óleo tenha algum efeito sobre a postura das teleóginas e a eclosão das larvas, contribuindo, dessa forma, para a diminuição da reinfestação dos animais. 
TABELA 1. Número de teleóginas por animal com a utilização de óleo de citronela (Cymbopogon nardus $\mathrm{L}$. Rendle) e do amitraz no controle do carrapato [Rhipicephalus (Boophilus) microplus] em bovinos da raça Holandês malhada de preto. Santa Maria, RS, 2007.

\begin{tabular}{|c|c|c|c|c|}
\hline \multirow{2}{*}{ Avaliações } & \multicolumn{3}{|c|}{ Teleóginas ( $\mathrm{n}^{\circ}$ ) } & \multirow[b]{2}{*}{ CV $(\%)$} \\
\hline & Testemunha ${ }^{1}$ & Citronela $4 \%^{2}$ & Amitraz $^{3}$ & \\
\hline Pré-tratamento ${ }^{4: 5}$ & $13,5^{\mathrm{Bc}}$ & $38,2^{\mathrm{Aa}}$ & $29,0^{A b}$ & 12,61 \\
\hline \multicolumn{5}{|l|}{ Pós-tratamento } \\
\hline $7^{-} \mathrm{dia}^{4}$ & $24,7^{\mathrm{Ba}}$ & $24,7^{\mathrm{BCa}}$ & $0,0^{\mathrm{Bb}}$ & 17,55 \\
\hline $14^{-} \mathrm{dia}^{4}$ & $59,5^{\mathrm{Ba}}$ & $33,7^{\mathrm{Bb}}$ & $2,5^{\mathrm{BC}}$ & 20,14 \\
\hline $21^{\circ} \mathrm{dia}^{4}$ & $73,5^{\mathrm{Aa}}$ & $25,2^{\mathrm{BCb}}$ & $1,2^{\mathrm{BC}}$ & 15,63 \\
\hline $28^{0} \mathrm{dia}^{4,5}$ & $71,7^{\mathrm{Aa}}$ & $22,0^{c b}$ & $29,0^{\mathrm{Ab}}$ & 16,55 \\
\hline \multirow[t]{2}{*}{ CV (\%) } & 17,08 & 8,90 & 13,78 & \\
\hline & \multicolumn{4}{|c|}{ Eficácia no controle de teleóginas (\%) } \\
\hline Pré-tratamento ${ }^{4: 5}$ & 0,0 & $0,0^{B}$ & $0,0^{\mathrm{C}}$ & 0,00 \\
\hline \multicolumn{5}{|l|}{ Pós-tratamento } \\
\hline $7^{-} \mathrm{dia}^{4}$ & $0,0^{\circ}$ & $35,3^{A b}$ & $100,0^{4 a}$ & 15,68 \\
\hline $14^{\circ} \mathrm{dia}^{4}$ & $0,0^{c}$ & $11,8^{A b}$ & $91,0^{\mathrm{Ba}}$ & 15,60 \\
\hline $21^{\circ} \mathrm{dia}^{4}$ & $0,0^{c}$ & $34,0^{A b}$ & $95,2^{\mathrm{Ba}}$ & 10,68 \\
\hline $28^{0} \mathrm{dia}^{4: 5}$ & $0,0^{b}$ & $42,4^{\mathrm{Aa}}$ & $0,0^{\mathrm{Bb}}$ & 23,99 \\
\hline$C V(\%)$ & 0,00 & 30,42 & 13,86 & \\
\hline
\end{tabular}

A В С Médias com letras distintas, na coluna, indicam diferença significativa $(p<0,05)$ pelo teste de Bonferroni; a b $\mathrm{c}$ Médias com letras distintas, na linha, indicam diferença significativa $(p<0,05)$ pelo teste de Bonferroni; ${ }^{1}$ Testemunha = grupo controle negativo; ${ }^{2} \mathrm{Citronela}$ $4 \%=$ óleo de citronela a $4 \%+0,5 \%$ de detergente neutro; ${ }^{3} \mathrm{Amitraz}=$ a $0,025 \%$, grupo controle positivo; ${ }^{4} \mathrm{Banho}$ com óleo de citronela a $4 \%+0,5 \%$ de detergente neutro; ${ }^{5}$ Banho com amitraz a $0,025 \%$.

Para mosca-dos-chifres (Tabela 2), observase que houve baixa eficácia de controle, verificada apenas no 70 dia de avaliação. Para mosca-dosestábulos e mosca doméstica houve comportamento similar ao da mosca-dos-chifres, considerando o mesmo período de avaliação. Comparativamente, observa-se que, embora baixa, o óleo de citronela apresentou eficácia de controle de mosca-dosestábulos e mosca doméstica em todas as avaliações.

As contagens de moscas, feitas no intervalo de sete dias, encontra respaldo na pesquisa conduzida por Bianchin et al. (2006) devido à duração do ciclo biológico da mosca-dos-chifres que varia de duas a três semanas. Outra observação importante é quanto ao número de moscas, observado no prétratamento (média de 10,1 e 14,2 para mosca-doschifres e mosca-dos-estábulos + mosca doméstica), que é bem inferior às infestações relatadas em outras pesquisas conduzidas no RS, com média de 84 moscas entre março e maio (Martins, 2006), e em outras regiões do País, sendo verificadas infestações com 80 (Bianchim \& Alves, 2002) e 85 moscas/ animal (Barros, 2001). Assim, a baixa infestação verificada no presente trabalho pode ter dificultado a análise dos dados, considerando o comportamento desses dípteros diante de diferentes condições antrópicas e ambientais (Axtell, 1986).

Com relação às amostras de sangue (Tabela 3), foram observadas reduções do número de hemácias, com conseqüente aumento do volume corpuscular médio e valores normais da concentração de hemoglobina corpuscular média. Esses resultados indicam que os animais apresentam anemia macrocítica normocrômica (Feldman et al., 2000). O comportamento desses parâmetros foi, provavelmente, decorrente do efeito que o carrapato provoca ao alimentar-se de sangue do animal (Johnston \& Haydock, 1969).

Os valores maiores do volume corpuscular médio refletem indiretamente a presença de reticulócitos no sangue, revelando que a anemia é regenerativa. Estas alterações foram observadas em todos os momentos de coleta, devendo-se considerar que os animais apresentaram infestações variadas 

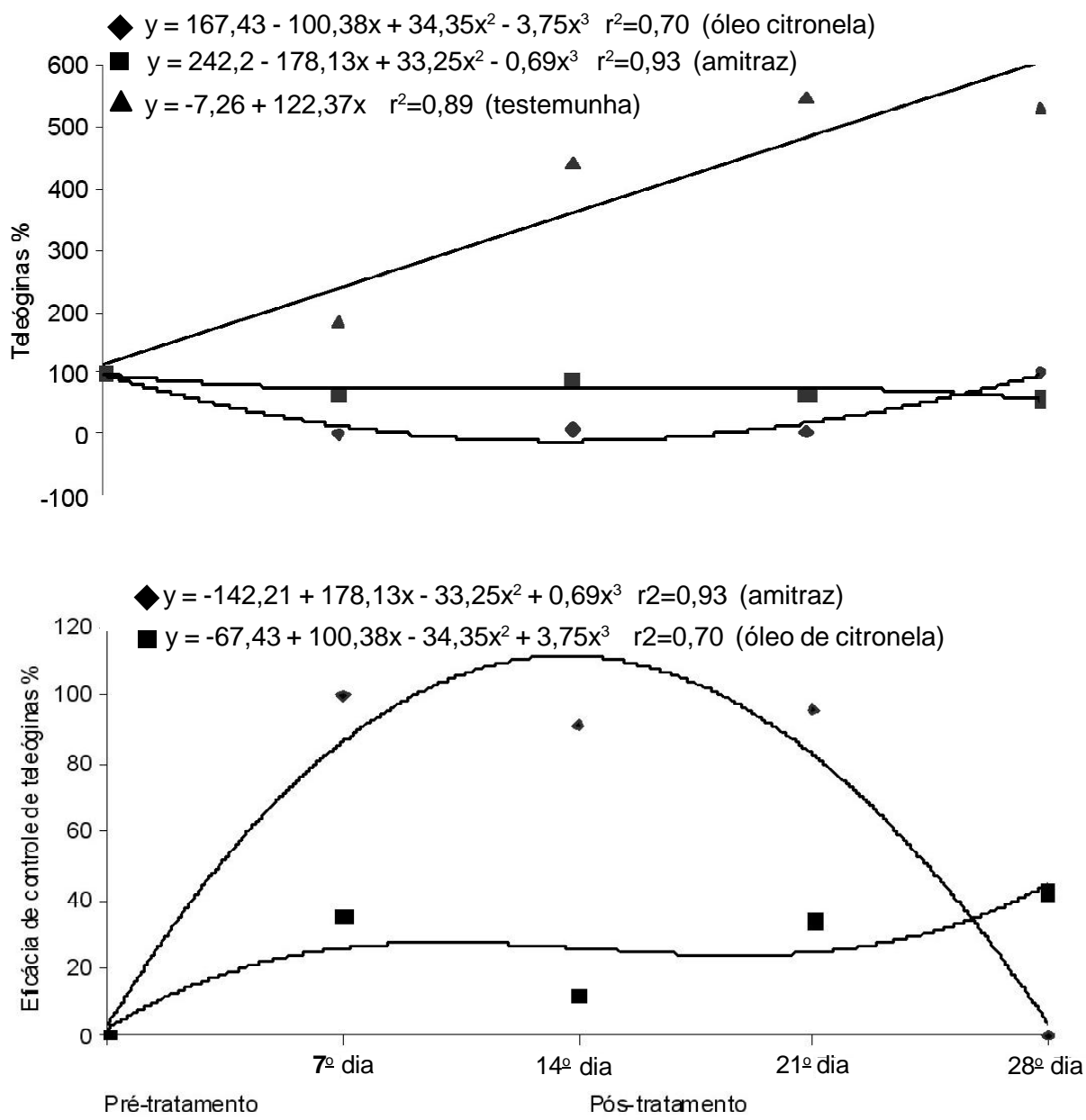

FIGURA 1. Formulações com óleo de citronela [Cymbopogon nardus (L.) Rendle] a $4 \%+0,5 \%$ de detergente neutro e amitraz a $0,025 \%$ no controle de teleóginas de bovinos [Rhipicephalus (Boophilus) microplus]. Santa Maria, RS, 2007

TABELA 3. Parâmetros sanguíneos médios de amostras coletadas antes e após a aplicação dos tratamentos (amitraz a 0,025\%; óleo de citronela a 4\%) em vacas lactantes da raça Holandês malhada de preto. Santa Maria, RS, 2007.

\begin{tabular}{|c|c|c|c|c|c|c|c|}
\hline \multirow[t]{3}{*}{ Parâmetros } & \multicolumn{6}{|c|}{ Tratamentos } & \multirow[t]{3}{*}{ Padrão ${ }^{2}$} \\
\hline & \multicolumn{2}{|c|}{ Testemunha } & \multicolumn{2}{|c|}{ Citronela } & \multicolumn{2}{|c|}{ Amitraz } & \\
\hline & Antes & Após $^{1}$ & Antes & Após $^{1}$ & Antes & Após $^{1}$ & \\
\hline Hemáceas $\left(10^{6}\right)$ & 3,7 & 3,6 & 4,5 & 4,2 & 4,7 & 4,47 & $5-10$ \\
\hline Hematócrito (\%) & 22 & 23 & 26,5 & 25,6 & 26,5 & 27,7 & $25-40$ \\
\hline Hemoglobina $\left(\mathrm{g} \mathrm{dL}^{-1}\right)$ & 7,7 & 8,4 & 9,8 & 9,27 & 9,8 & 9,8 & $8-15$ \\
\hline VCM (fl) & 59 & 63,2 & 60,2 & 60,7 & 55,5 & 61,7 & $40-60$ \\
\hline $\mathrm{CHCM}(\%)$ & 35 & 36,4 & 37,1 & 36 & 36,9 & 35,3 & $30-36$ \\
\hline Leucócitos & 16100 & 16375 & 16975 & 17562 & 24150 & 25950 & $4000-12000$ \\
\hline Neutófilos $(/ \mu \mathrm{L})$ & 4446 & 2762 & 3760 & 4701 & 6206 & 5451 & $600-4000$ \\
\hline Linfócitos $(/ \mu \mathrm{L})$ & 10085 & 12035 & 11685 & 11011 & 15910 & 17628 & $2500-7000$ \\
\hline Bastonetes $(/ \mu \mathrm{L})$ & 0 & 0 & 0 & 40 & 118 & 0 & $0-120$ \\
\hline Eosinófilos $(/ \mu \mathrm{L})$ & 1463 & 1053 & 1175 & 1247 & 1309 & 1403 & $0-2400$ \\
\hline Monócitos $(/ \mu \mathrm{L})$ & 105 & 524 & 334 & 560 & 606 & 1466 & $25-840$ \\
\hline
\end{tabular}

Dados não submetidos à análise estatística; $\mathrm{VCM}$ = volume corpuscular médio; $\mathrm{CHCM}=$ concentração de hemoglobina corpuscular média; 'Valor médio de amostras coletadas às 72 e 120 horas após a aplicação dos tratamentos; ${ }^{2 B}$ Bovinos adultos. 
no mês anterior a presente avaliação. Os valores de leucócitos, encontrados estão acima do padrão de normalidade para a espécie bovina, situados entre 4.000 a 12.000 (Duncan \& Prasse, 1982). Os mesmos autores afirmaram que taxas aumentadas de leucócitos são comuns em processos parasitários de evolução crônica. Em áreas tropicais e subtropicais, quando os animais estão constantemente expostos às infestações por carrapatos, ocorre pré-munição contínua nos rebanhos, determinando quadro de leucocitose nos animais pré-munidos (Birgel et al., 1995).

Assim, conclui-se que a solução contendo $4 \%$ de óleo de citronela, aplicada estrategicamente a cada sete dias, controlou a infestação de carrapatos. No período avaliado encontrou-se uma relação de 1:2,5 para o amitraz e o óleo de citronela, respectivamente, para controlar a infestação. Para mosca-dos-chifres, mosca-dos-estábulos e mosca doméstica o controle com o produto fitoterápico foi baixo. Os dados do hemograma demonstram similaridades entre os grupos avaliados.

\section{REFERÊNCIA}

AXTELL, R.C. Fly control in confined livestock and poultry production. Greensboro: CIBA-GEIGY, 1986. 59p. BARROS A.T.M. Dynamics of Haematobia Irritans irritans (Diptera: Muscidae) infestation on Nelore cattle in the Pantanal, Brasil. Memórias do Instituto Oswaldo Cruz, v.96, n.4, p.445-50, 2001.

BIANCHIN, I.; ALVES, R.G.O. Mosca-dos-chifres, Haematobia irritans: comportamento e danos em vacas e bezerros Nelore antes da desmama. Pesquisa Veterinária Brasileira, v.22, n.3, p.109-13, 2002.

BIANCHIN, I. et al. Eficiência do pó de alho (Allium sativum) no controle dos parasitos de bovinos. Campo Grande: Embrapa Gado de Corte, 1999. 31p. (Boletim de pesquisa, 8).

BIANCHIN, I. et al. Sazonalidade de Haematobia irritans no Brasil Central. Pesquisa Veterinária Brasileira, v.26, n.2, p.79-86, 2006.

BIRGEL, E.H. et al. Prevalência da infecção pelo vírus leucose dos bovinos, em animais da raça Jersey, criados no Estado de São Paulo, Pesquisa Veterinária Brasileira, v.15, n.4, p.93-9, 1995.

CAMILLO, G. et al. Eficiência in vitro de acaricidas sobre carrapatos de bovinos no Estado do Rio Grande do Sul, Brasil. Ciência Rural, v.39, n.2, p.490-5, 2009.

CASTREJÓN, F.M. et al. Repellence of Boophilus microplus larvae in Stylosanthes humilis and Stylosanthes hamata plants. Parasitologia Latinoamericana, v.58, n.23, p.118-21, 2003.

CHAGAS, A.C.S. et al. Efeito acaricida de óleos essenciais e concentrados emulsionáveis de Eucalyptus spp em Boophilus microplus. Brazilian Journal of
Veterinary Research and Animal Science, v.39, n.5, p.247-53, 2002.

CHAGAS, A.C.S. et al. Sensibilidade do carrapato Boophilus microplus a solventes. Ciência Rural, v.33, n.1, p.109-14, 2003.

CHUNGSAMARNYART, N.; JIWAJINDA, S. Acaricidal activity of volatile oil from lemon and citronella grasses on tropical cattle ticks. Kasetsart Journal (Natural Science), v.26, n.5, p.46-51, 1992.

CORDOVÉS, C.O. Carrapato: controle ou erradicação. Guaíba: Agropecuária, 1997. 176p.

DUBOIS, R. Pesticidas, antibióticos e a intoxicação humana. A Hora Veterinária, v.13, n.72, p.55-60, 1993. DUNCAN, J.R.; PRASSE, K.W. Patologia clínica veterinária. Rio de Janeiro: Guanabara Koogan, 1982. $217 p$.

FELDMAN, B.C. et al. Veterinary hematology. Philadelphia: Lippincott Williams \& Wilkins, 2000. 1344p. GRISI, L. et al. Impacto econômico das principais ectoparasitoses em bovinos no Brasil. A Hora Veterinária, v.21, n.125, p.8-10, 2002.

HEIMERDINGER, A. et al. Extrato alcoólico de capimcidreira no controle do Boophilus microplus em bovinos. Revista Brasileira de Parasitologia Veterinária, v.15, n.1, p.37-9, 2006.

JOHNSTON, L.A.Y.; HAYDOCK, K.P. The effect of cattle tick (Boophilus microplus) on production of Brahmancross and British-breed cattle in northern Australia. Australian Veterinary Journal, v.45, n.4, p.175-9, 1969. MARTINS, R.M. Estudo in vitro da ação acaricida do óleo essencial da gramínea Citronela de Java (Cymbopogon winterianus Jowitt) no carrapato Boophilus microplus. Revista Brasileira de Plantas Medicinais, v.8, n.2, p.718, 2006.

MEKONNEN, S. In vivo evaluation of amitraz against ticks under field conditions in Ethiopia. Journal of the South African Veterinary Association, v.72, n.1, p.44-5, 2001. OLIVEIRA, A.A.; AZEVEDO, H.C. Resistência do carrapato Boophilus microplus a carrapaticidas em bovinos de leite na região dos tabuleiros costeiros de Sergipe. Revista Científica Rural, v.7, n.2, p.64-71, 2002.

OLIVO, C.J. et al. Óleo de citronela no controle do carrapato de bovinos. Ciência Rural, v.38, n.2, p.406-10, 2008.

RADOSTITS, O.M. et al. Clínica veterinária: um tratado de doenças dos bovinos, ovinos, suínos caprinos e eqüinos. Rio de Janeiro: Guanabara Koogan, 2002. 1737p.

RAJA, N. et al. Effect of volatile oils in protecting stored Vigna unguiculata (L.) Walpers against Callosobruchus maculatus (F.) (Coleóptera: Bruchidae) infestation. Journal of Stored Products Research, v.37, n.2, p.127-32, 2001. SHASANY, A.K. et al. Phenotypic and RAPD diversity among Cymbopogon Winterianus Jowitt accessions in relation to Cymbopogon nardus Rendle. Genetic Resources and Crop Evolution, v.47, n.5, p.553-9, 2000.

WHARTON, R.H. et al. Assessment of the efficiency of acaricides and their mode of aplication against the cattle tick Boophilus microplus. Australian Journal of Agricultural Research, v.21, n.5, p.985-1006, 1970. 